\title{
Review of: "Effect of electroencephalography-guided anesthesia on neurocognitive disorders in elderly patients undergoing major non-cardiac surgery: A trial protocol The POEGEA trial (POncd Elderly GEneral Anesthesia)"
}

Thepakorn Sathitkarnmanee

Potential competing interests: The author(s) declared that no potential competing interests exist.

This trial protocol was well prepared. The Introduction provided adequate rational background leading to this study. The Objectives covers all relevant outcomes at different timepoints.

The Materials and method provides details of every step which is reproduceable. However, I have a few comments with the Hemodynamic management protocol. Since the major factor affecting neurocognitive disorder is cerebral blood flow, the cerebral blood flow must be optimized at same level. Preload optimization is the fundamental. The protocol uses pulse pressure (PPV) variation as indicator for fluid bolus titration but lacks details. The exact value of PPV to be maintained must be stated to ensure that fluid administration will not be at the discretion of the anesthesiologist in charge. Vasopressor administration should be considered after preload optimization. Another comment is that the protocol gives detail of phenylephrine dosage to maintain MAP of $+/-20 \%$, what is the intervention in case MAP > $20 \% ?$ 